including tumour-necrosis factor (TNF)- $\alpha$ and members of the IL-19 family (IL-19, IL20 and IL-24) ${ }^{3}$. As IL-23 (ref. 11) and these other cytokines are also present at elevated levels within psoriatic plaques, it is noteworthy that the IL-23-induced epidermal response was dependent on the presence of TNF- $\alpha$ as well as the IL-20R2 receptor, which is shared by IL-19 family members. Integrating these new results into a cytokine network model for the immunopathogenesis of psoriasis reveals a remarkably reciprocal exchange of signals between epidermal keratinocytes and immunocytes (Fig. 1).

The new findings build on previous work turning attention to a cytokine networking theory. The early phases of psoriasis research focused on identifying a high-affinity foreign antigen that may have been derived from an infectious agent to drive distinct cell clones in a classical adaptive immune response. But no clonal T-cell expansion was consistently identified, and, together with other develop-

ments, prompted the field to move in another direction. Researchers began to think about innate immunity contributing to an inappropriate local tissue inflammatory reaction in psoriasis. Along this line, it should be noted that IL-23 has been implicated in local mucosal immunopathology by means of innate immune mechanisms ${ }^{12}$

By taking a fresh look at the local cytokine milieu within psoriatic plaques, analyses of human tissue samples revealed that IL-23 and IL-12 share a common subunit, termed p40 (ref. 11); previously, p40 was thought to be exclusive to IL-12. The findings led to reevaluation of experiments targeting p40 and to initial suggestions that IL-23 may be more important in psoriasis immunopathogenesis than IL-12-thereby implying that the initial classification of psoriasis as a purely $\mathrm{T}_{\mathrm{H}} 1$ type disease may require modification to take into account a role for $\mathrm{T}_{\mathrm{H}} 17 \mathrm{~T}$ cells.

Despite the impressive and complementary data of Zheng et al. ${ }^{2}$ and Chan et al. ${ }^{3}$, there are still a few caveats to consider. First, it must be acknowledged that psoriatic lesions in humans have distinctive clinical, histological and immunopathological features, not all of which are entirely reproduced in mouse skin ${ }^{13}$.

Second, as the field moves forward, it is important to remember that bona fide psoriatic plaques can be entirely reversed, and such healing occurs without scar formation. Thus, pathways by which the IL-23- and $\mathrm{T}_{\mathrm{H}} 17$-mediated inflammation is terminated must take into consideration these clinical observations.

Third, despite the persistent and often prominent inflammatory milieu within and around plaques, only rarely do these lesions progress to malignancy or harbor the development of nonepithelial malignancies such as cutaneous melanoma. As IL-23 has been found to promote tumor incidence and growth, this paradox is yet to be resolved ${ }^{14}$. Last, but not least, it is unclear what triggers the production of IL-23 by sentinel cells, such as dendritic cells and macrophages in symptomless skin, thereby initiating the cytokine-driven tissue response in genetically susceptible individuals. Theories for the immunopathogenesis of psoriasis must account for these salient, and sometimes overlooked, phenotypic characteristics of lesions.

Despite these points of concern, the beginning of 2007 is shaping up as one of the most remarkable years for investigative skin biolo- gists. Yet, much work remains to be done to determine how IL-23 operates in psoriasis. It is no easy feat for the body to create a psoriatic plaque - and it is not easy for us to begin to understand how to reverse it.

Future work must take into account the many IL-17 isoforms and include a complete evaluation of direct and indirect effects of IL-23 on resident and recruited cell types involved in remodeling the skin. Understanding how genes that encode for cytokine receptors influence the responsiveness of immunocytes and keratinocytesincluding complex interactions mediated by $\mathrm{T}_{\mathrm{H}} 1$ and $\mathrm{T}_{\mathrm{H}} 17$ pathways - are likely to pay dividends beyond understanding the skin and psoriasis to diseases that affect other organ systems.

\section{COMPETING INTERESTS STATEMENT}

The author declares that he has no competing financial interests.

1. Steinman, L. Nat. Med. 13, 139-145 (2007).

2. Zheng, Y. et al. Nature 445, 648-651 (2007).

3. Chan, J.R. et al. J. Exp. Med. 203, 2577-2587 (2006).

4. Nickoloff, B.J. Arch. Dermatol. 127, 871-884 (1991).

5. Duerr, R.H. et al. Science 314, 1461-1463 (2006).

6. Cargill, M. et al. Am. J. Hum. Genet. 80, 273-390 (2007).

7. Iwakura, Y. \& Ishigame, H. J. Clin. Invest. 116 1218-1222 (2006)

8. Boniface, K. et al. J. Immunol. 174, 3695-3702 (2005).

9. Sano, S. et al. Nat. Med. 11, 43-49 (2005).

10. Wolk, K. et al. Eur. J. Immunol. 36, 1309-1323 (2006).

11. Lee, E. et al. J. Exp. Med. 199, 125-130 (2004).

12. Uhlig, H.H. et al. Immunity 25, 309-318 (2006).

13. Nickoloff, B.J. Trends Mol. Med. 12, 102-106 (2006).

14. Langowski, J.L. et al. Nature 442, 461-465 (2006)

\title{
Skin safety
}

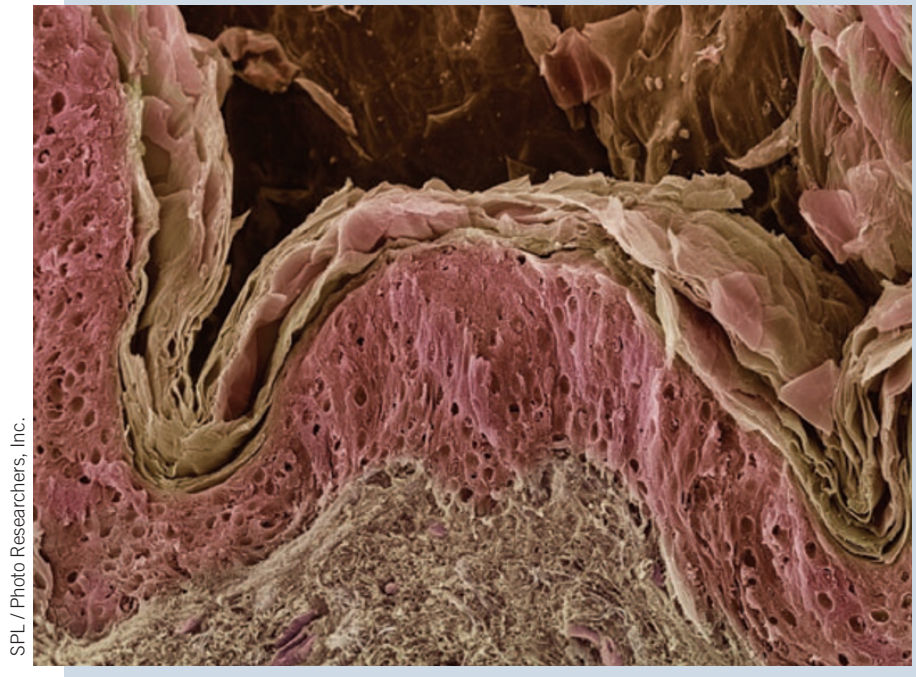

Healthy skin.
While basic researchers tease out the critical cytokines behind psoriasis, clinicians have not been idle. A monoclonal antibody that recognizes both IL-12 and IL-23 has been making its way through clinical trials; the most recent results were reported February 8 in the New England Journal of Medicine (356, 580-592).

Gerald Krueger et al. treated 320 individuals with psoriasis with either a placebo or a monoclonal antibody that binds the p40 subunit shared by both IL-12 and IL-23. The researchers report a 75 percent improvement in psoriasis symptoms in about half of subjects at the lowest doses and about 80 percent of subjects receiving the highest doses. A higher percentage of treated individuals suffered severe effects such as cancer or a heart attack—but the effects were not significant and larger trials are needed.

The study does nothing to resolve whether IL-12 or IL-23 is the most effective target for psoriasis. But the researchers point out that the efficacy is similar to that of other biologic therapeutics on the market, such as etanercept, which blocks TNF- $\alpha$ signaling. -Charlotte Schubert 